\title{
Hausärzte und kein Nachwuchs - Ursachen aus erster Hand
}

\section{Jörg Fritschi}

Die weibliche Form schliesst die männliche ein und umgekehrt.
Korrespondenz:

Dr. med. Jörg Fritschi Klein Buholz 3

CH-6012 Obernau Tel. 0413201055 Fax 0413201175

j.fritschi@hin.ch
In der Schweiz ist ein Hausarztmangel absehbar. Erstmals wurden die Motive der Karrierewahl von Jungärztinnen und -ärzten wissenschaftlich untersucht. Auf Anregung des Dachverbandes der Schweizer Ärztenetzwerke med-swiss.net hat die Abteilung für psychosoziale Medizin des Universitätsspitals Zürich in einer prospektiven Studie Assistenzärztinnen und -ärzte befragt. Die wahren Ursachen des sich abzeichnenden Hausärztemangels überraschen. Die dringend notwendigen Gegenmassnahmen erfordern ein entschlossenes Handeln der Politiker, der Standespolitiker und der Versicherer.

Unter der Diskussionsleitung von Hans Fahrländer, Chefredaktor der Aargauer Zeitung, engagierten sich im August 2007 im Universitätsspital Zürich die folgenden Personen auf dem Podium:

- PD Dr. phil. Richard Klaghofer, Abteilung psychosoziale Medizin, Universitätsspital Zürich, Forschungsprojektgruppe;

- Dr. med. Franz Marty, Facharzt für Allgemeinmedizin FMH, Forschungsprojektgruppe;

- Dr. med. vet. Markus Dürr, Präsident der Gesundheitsdirektorenkonferenz;

- lic. iur. Nadine Facchinetti, Bundesamt für Gesundheit (BAG);

- Prof. Dr. med. Martin Täuber, Dekan der Medizinischen Fakultät der Universität Bern;

- Fritz Britt, Fürsprecher, Direktor santésuisse;

- Dr. med. Philip Dreiding, Facharzt für Allgemeinmedizin FMH, Vorstand med-swiss.net.

\section{Kernaussagen der Studie}

Die Einkommensunsicherheit des Hausarztberufes spricht auch für die inskünftigen Hausärztinnen am meisten gegen die eingeschlagene Karriere.

Extraprofessionelle Belange, z. B. Familie, haben für die zukünftigen Hausärztinnen einen hohen Stellenwert.

Nur 17\% der befragten 504 Assistenzärztinnen und Assistenzärzte entscheiden sich für den Hausarztberuf. 91\% der befragten zukünftigen Hausärztinnen möchten in einer Gruppenpraxis arbeiten.

\section{Voten aus der Podiumsdiskussion}

Das Studium (Ausbildung) und die anschliessenden Assistenzjahre (Weiterbildung) sind für die Karrierewahl entscheidend.
Der Hausarzt ist kein gescheiterter Spezialist, sondern dank seines breiten Wissens befähigt, 9 von 10 ambulanten Patienten selbständig und abschliessend zu behandeln. Die Hausärztin ist die Spezialistin für alle Fälle, gerade auch für polymorbide Patienten.

Die berufliche Selbständigkeit ist heute offenbar nicht mehr attraktiv.

Nur gutausgebildete Grundversorger/innen garantieren für einen qualitativ hochstehenden und damit auch kostengünstigen ärztlichen Notfalldienst.

\section{Massnahmen und Lösungsideen}

Für zukünftige Hausärztinnen stehen während der Assistenzjahre Ausbildungsplätze zur Verfügung, die auf die Belange einer Familie Rücksicht nehmen.

Eine im Vergleich zu anderen akademischen Berufen angemessene Entschädigung der Hausärzte ist zwingend nötig, insbesondere im Rahmen des Notfalldienstes.

Das Image der Hausärztin muss auf allen Ebenen gefördert werden. Nicht nur die Hausärzte selber sind bemüht, ein positives Bild ihres Berufsstandes zu vermitteln. Auch die standespolitischen Organisationen sind aufgerufen, die Hausarztmedizin als eigenständige und vollwertige Disziplin anzuerkennen. Die Politiker sprechen nicht nur darüber, wie wichtig und wertvoll die Hausarztmedizin sei, sondern setzen klare Zeichen zur Förderung der Grundversorgung.

Alle Medizinstudenten absolvieren ein Praktikum in einer Hausarztpraxis.

Die Weiterbildung in der Praxisassistenz ist durch alle Kantone intensiv zu fördern.

Der Praxiszulassungsstopp muss für Grundversorger gelockert werden; er schreckt von der Hausarztkarriere ab.

Das KVG bedarf einer dringlichen Revision (Risikoausgleich, monistische Spitalfinanzierung, Förderung von Managed Care).

Gesundheitspolitische Gremien entscheiden inskünftig nicht über, sondern mit den Hausärztinnen und Hausärzten zusammen.

Wer nimmt die Massnahmen in die Hand? Politiker, Versicherer und die Ärzteschaft sind gleichermassen aufgerufen. Nur mit einer partnerschaftlichen Zusammenarbeit kann dem Hausärztemangel nachhaltig begegnet werden. 